\title{
Adrenoceptor Responses in Human Embryonic Stem Cell-Derived Cardiomyocytes: a Special Focus on Electrophysiological Property
}

\author{
Weiwei Jiang, ${ }^{1}$ Xingjian $\mathrm{Hu},{ }^{1}$ Fei Li, Geng Li, and Yin Wang \\ Departments of Cardiovascular Surgery (X.H., F.L., G.L., Y.W.) and Gastroenterology (W.J.), Union Hospital, Tongji Medical \\ College, Huazhong University of Science and Technology, Wuhan, China; and Department of Biomedical Engineering, The Johns \\ Hopkins University, Baltimore, Maryland (Y.W.)
}

Received February 11, 2020; accepted March 19, 2020

\begin{abstract}
Human embryonic stem cell-derived cardiomyocytes (hESC-CMs) have become a promising cell source for cardiovascular research. The electrophysiological characteristic of hESC-CMs has been generally studied, but little is known about electrophysiological response to adrenergic receptor (AR) activation. This study aims to characterize electrophysiological response of hESC-CMs to adrenergic stimulation in terms of the conduction velocity (CV) and action potential (AP) shape. The H9 hESC-CMs were acquired by a classic differentiation protocol and cultured to achieve confluent cell monolayers. The AP shape and CV among the monolayers were recorded using optical mapping during electrophysiological and pharmacological stimulation experiments. Quantitative realtime polymerase chain reaction and Western blot were adopted to determine the expression levels of Connexin and ion channel gene and protein. Chronic $\beta$-AR stimulation by isoproterenol for 24 hours in hESC-CM monolayers increased CV by approximately $50 \%$, whereas $\alpha$-AR or acute $\beta$-AR stimulation had no significant effect; chronic $\beta$-AR stimulation resulted in a significant Connexin (Cx) 43 and $\mathrm{Na}_{\mathrm{v} 1.5}$ upregulation at both protein and mRNA level. Isoproterenol-induced CV accelerating and $\mathrm{Cx} 43$ and $\mathrm{Na}_{\mathrm{v} 1.5}$ upregulation in hESC-CMs, which was attenuated by selective $\beta 1$-adrenoceptor antagonist CGP 20712A but not selective $\beta 2$-antagonist ICI 118551. Moreover, pretreatment with protein
\end{abstract}

kinase $A(P K A)$ inhibitor H89, mitogen-activated protein kinase (MAPK)/extracellular signal-regulated kinase (MEK) inhibitor SB203580, and MAPK inhibitor PD98059 suppressed the isoproterenol-induced CV accelerating and $\mathrm{Cx} 43$ upregulation, whereas it had no significant effect on $\mathrm{Na}_{\mathrm{v} 1.5}$ upregulation. The AP shape in hESC-CM monolayers was less susceptible by either $\beta$-AR or $\alpha$-AR stimulation. It was $\beta 1$ AR not $\beta 2$-AR contributing to the modification of conduction velocity among hESC-CM monolayers. Chronic $\beta 1$-AR stimulation accelerates $\mathrm{CV}$ by upregulating $\mathrm{Cx} 43$ via PKA/MEK/ MAPK pathway.

\section{SIGNIFICANCE STATEMENT}

These data provide new insight into the electrophysiological characteristics of human embryonic stem cell-derived cardiomyocytes (hESC-CMs) and depict a concise signaling pathway in the adrenergic receptor (AR) regulation of action potential shape and electrical propagation across hESC-CM monolayer. It is $\beta 1$-AR not $\beta 2-A R$ contributing to the modification of conduction velocity in $\mathrm{hESC}-\mathrm{CMs}$ and accelerating conduction velocity by upregulating Connexin 43 via protein kinase $A /$ mitogenactivated protein kinase (MAPK)-extracellular signal-regulated kinase/MAPK pathway.

\section{Introduction}

Human pluripotent stem cell-derived cardiomyocytes (hPSC$\mathrm{CMs}$ ), including induced human pluripotent and human embryonic stem cell-derived cardiomyocytes (hESC-CMs), have become an important cell resource for cardiac regeneration, drug screening, and models of cardiovascular disease (Dakhore et al., 2018; Protze et al., 2019; Samak and Hinkel,

This study was financially supported by Natural Science Foundation of China (81700317, 81400290, and 81670351).

${ }^{1}$ W.J. and X.H. contributed equally to the work.

https://doi.org/10.1124/jpet.120.265686.
2019). What is particularly crucial for the usage of hPSC-CMs is a profound understanding of their electrophysiological properties (Liu et al., 2016). The electrophysiological characteristics of hPSC-CMs have been investigated by several studies, most of which focused on the properties of specific ion transporters, including sodium current $\left(\mathrm{I}_{\mathrm{Na}}\right)$, calcium current, potassium current $\left(\mathrm{I}_{\mathrm{K}}\right)$, and so on, whereas the relationship between adrenergic receptor (AR) signaling and electrophysiological characteristics of hPSC-CMs has not yet been revealed (van den Heuvel et al., 2014; Wang et al., 2019).

As a canonical signaling pathway for the cardiomyocyte, the stimulation of AR system increases beating rate, force of

ABBREVIATIONS: AP, action potential; $\mathrm{APD}, \mathrm{AP}$ duration; $\mathrm{APD}_{30}, \mathrm{APD}$ at $30 \%$ repolarization; $\mathrm{AR}$, adrenergic receptor; $\mathrm{CV}$, conduction velocity; $\mathrm{Cx}$, Connexin; $\mathrm{dd}_{2} \mathrm{O}$, double distilled $\mathrm{H}_{2} \mathrm{O}$; GAPDH, glyceraldehyde-3-phosphate dehydrogenase; GJA1, gap junction alpha-1; hESC, human embryonic stem cell; hESC-CM, hESC-derived cardiomyocyte; hPSC-CM, human pluripotent stem cell-derived cardiomyocyte; I $_{\mathrm{Na}}$, sodium current; ISO, isoproterenol; KCNQ1, potassium voltage-gated channel subfamily Q member 1; MAPK, mitogen-activated protein kinase; MEK, MAPK/ extracellular signal-regulated kinase; PCR, polymerase chain reaction; PKA, protein kinase A; qRT-PCR, quantitative real-time PCR; SCN5A, sodium voltage-gated channel alpha subunit 5. 
contraction, and velocity of contraction/relaxation in adult cardiomyocytes (Campbell et al., 2014). Although attention has been given primarily to the regulatory mechanisms of AR signaling on the beating rate and contractility, few studies explored the effect of AR signaling on the electrophysiological characters and electrical propagation among hPSC-CMs (Brito-Martins et al., 2008; Pillekamp et al., 2012). Little is known about electrophysiological response of hPSC-CMs to adrenergic adrenoreceptor, and the effect of $\mathrm{AR}$ activation on action potential (AP) and conduction velocity (CV) is unclear (Veeraraghavan et al., 2014).

Thus, the present study aims to 1) culture hESC-CMs to achieve confluent monolayers and perform electrophysiological and pharmacological studies using optical mapping method, 2) evaluate the alteration in APDs and average CV among the hESC-CM monolayer after $\alpha$ - or $\beta$-AR stimulation, and 3) identify the possible adrenoceptor receptor subtype(s) and mechanisms involved in this process.

\section{Materials and Methods}

Human Embronic Stem Cell Culture and Differentiation. We cultured H9 human embryonic stem cells (hESCs) in a monolayerbased protocol (Bhattacharya et al., 2014). To be short, undifferentiated hESCs were cultured in Geltrex (Thermofisher Scientific, Waltham, MA)-coated tissue culture plates and incubated in E8 media (Thermofisher Scientific) for 4 days before differentiation. Then, the cells were incubated in RPMI 1640 (Thermofisher Scientific) with B27 without insulin (Thermofisher Scientific) to begin the differentiation. Media were supplemented with $6 \mu \mathrm{M}$ CHIR99021 (Selleckchem, Houston, TX) for the first 48 hours and then $5 \mu$ M IWR-1 (SigmaAldrich, St. Louis, MO) for the following 48 hours. The media were then replaced with RPMI 1640 with B27 and insulin (Thermofisher Scientific) on day 9 of the differentiation. hESC-CM monolayers were harvested using $0.05 \%$ trypsin between 10 and 12 days of the differentiation and replated at a density of 250,000 cells $/ \mathrm{cm}^{2}$ to form a confluent monolayer, which was cultured for extra 30 days (day 40-45 postdifferentiation) for the experiments.

Pharmacological Experiments. Targets were first dissolved with either $\mathrm{ddH}_{2} \mathrm{O}$ or DMSO as the stock solution and then diluted to certain concentration with Tyrode's solution as the working solution. Isoproterenol (ISO) (Sigma-Aldrich) dissolved in $\mathrm{dd}_{2} \mathrm{O}$ to $1 \mathrm{mM}$ was used as the stock solution and then diluted to $1 \mu \mathrm{M}$ in Tyrode's solution when used. CGP 20712A (Tocris, Minneapolis, MN) was dissolved in $\mathrm{ddH}_{2} \mathrm{O}$ to $1 \mathrm{mM}$ as the stock solution and diluted to $0.3 \mu \mathrm{M}$ in Tyrode's solution when used. Epinephrine (Sigma-Aldrich) was dissolved with DMSO to $10 \mathrm{mM}$ and diluted to $10 \mu \mathrm{M}$ in Tyrode's solution. ICI 118551 (Tocris) was also dissolved in DMSO at $10 \mu \mathrm{M}$ as the stock solution and serially diluted to $50 \mathrm{nM}$ as the working solution. H89 (Sigma) was dissolved in DMSO at $1 \mathrm{M}$ as the stock solution and serially diluted to $10 \mathrm{mM}$ as the working solution. SB203580 (Tocris) was dissolved in DMSO at $1 \mathrm{mM}$ as the stock solution and diluted to $20 \mu \mathrm{M}$ as the working solution. PD98059 (Tocris) was dissolved in DMSO at $1 \mathrm{mM}$ as the stock solution and diluted to $20 \mu \mathrm{M}$ as the working solution.

Chronic adrenergic stimulation on the hESC-CM monolayers was performed by supplementation of $1 \mu \mathrm{mol}$ isoproterenol or $10 \mu \mathrm{mol} / \mathrm{L}$ epinephrine to the medium 24 hours before the experiments. Acute adrenergic stimulation was performed by supplementation of $1 \mu \mathrm{mol}$ isoproterenol or $10 \mu \mathrm{mol} / \mathrm{L}$ epinephrine 10 minutes before the experiments. For some experiments, the specific inhibitors were added 1 hour prior to chronic stimulation with ISO.

Electrophysiological Experiments. Optical mapping: hESC-CM monolayers $\left(250,000\right.$ cells $\left./ \mathrm{cm}^{2}\right)$ were first incubated in Tyrode's solution and stained with voltage-sensitive dye di-4-ANEPPS $(10 \mu \mathrm{M})$ (Sigma-Aldrich) for 10 minutes on a $35-\mathrm{mm}$ Petri dish. To eliminate cell motion and contraction interference, a 10- $\mu \mathrm{M}$ blebbistatin (SigmaAldrich) was applied. Point pacing was proceeded at least 10 minutes after blebbistatin was added. The experiments were conducted in a temperature controlled at $37^{\circ} \mathrm{C}$, and a $100 \times 100$-pixel CMOS camera (MiCAM Ultima-L, SciMedia, Costa Mesa, CA) was used for optical action potentials recording.

Customized MATLAB scripts were used for mapping data analysis. A $5 \times 5$ Gaussian filter were applied during optical recordings to remove excessively noisy background or low-signal channels. Activation maps were drawn according to the time of maximum AP upstroke rate. $\mathrm{CV}, \mathrm{AP}$ duration at $30 \%$, and $\mathrm{AP}$ duration at $80 \%$ were calculated from the optical voltage signal.

Western Blot Analysis. hESC-CMs were harvested and lysed with RIPA buffer (Thermofisher Scientific) cocktail supplemented with protease inhibitor (Sigma-Aldrich) and phosphatase inhibitor (Sigma-Aldrich). Total protein was quantified with a Pierce BCA kit (Thermofisher Scientific). A total protein lysate of $30 \mu \mathrm{g}$ was loaded for each lane on $4 \%-12 \%$ mini Tris-Bis gels (Bio-Rad, Hercules, CA) and transferred onto polyvinylidene difluoride membranes for immunoblotting. Membranes were blocked with nonfat milk and then incubated with primary antibodies for Kv11.1 (1:200, sc-377388; Santa Cruz), Kv7.1 (1:1000, sc-20816; Santa Cruz), Nav1.5 (1:200, asc-005; Alomone Laboratories), Connexin 43 (1:1000, C6219; Sigma), and GAPDH (1:1000, MAB374; EMD Millipore) overnight at $4^{\circ} \mathrm{C}$. The membrane was further incubated with secondary antibodies (1:20,000, goat anti-rabbit or mouse; LI-COR, Lincoln, NE) and imaged by Odyssey CLX (LI-COR) per the manufacturer's protocol. Detailed information of the antibodies was shown in Table 1. ImageJ software was used for a quantified and normalized protein intensities analysis.

Quantitative Real-Time Polymerase Chain Reaction. Quantitative real-time polymerase chain reaction (qRT-PCR) was performed on hESC-CM monolayers after mRNA was extracted as routine. Reverse transcription was performed to create cDNA with the Polymerase Chain Reaction (PCR) Master Mix kit (Thermo Fisher Scientific) using the MyGo Mini PCR system (IT-IS Life Science Ltd., Republic of Ireland). qRT-PCR was performed on each target in triplicate. Primer sequence of the PCR products were listed below: GJA1-sense, ACAGGTCTGAGTGCCTGAAC and GJA1-antisense, CGAAAGGCAGACTGCTCATC; SCN5A-sense, GTGCCCAGAAGC AGGATGAG, SCN5A-antisense, GGACATACAAGGCGTTGGTG; CACAN1C- sense, CACGGCTTCCTCGAATCTTG, CACAN1C- antisense, CTGTGGAGATGGTCGCATTG; KCNQ1-sense, CCGCCTGAACCGAGT AGAAG, KCNQ1-antisense, GTGTTGCTGGGCAGGAAGAG.

Statistical Methods. Data are presented as mean \pm S.E.M. Data were analyzed with Prism 7.0 (GraphPad Software, Inc.). Student's unpaired two-tailed $t$ test was used for two-group comparisons, and one-way ANOVA followed by Tukey's post hoc tests was conducted for multiple comparisons. $P<0.05$ was considered statistically significant.

\section{Result}

$\beta$-AR Stimulation on CV and APs in hESC-CM Monolayers. After being point stimulated at different pacing rates, the action potentials and their propagation across hESC-CM monolayers were recorded using optical mapping. As shown in Fig. 1, A and B, the CV was faster in hESC-CM monolayers stimulated with $1 \mu \mathrm{mol}$ ISO for 24 hours; when compared with the control monolayers $(11.2 \pm 0.3 \mathrm{~cm} / \mathrm{s}$ vs. $7.8 \pm 0.4 \mathrm{~cm} / \mathrm{s}$ at $1 \mathrm{~Hz}, P<0.001)$, the same results were observed in the other two different pacing rates $(0.5$ and $2 \mathrm{~Hz})$. Next, we investigated the impact of chronic $\beta$-adrenergic receptor stimulation on action potentials in hESC-CM monolayers; Fig. 1C shows the recorded APs in control and chronic ISO treatment monolayers at standard $1-\mathrm{Hz}$ pacing rate. As shown in Fig. 1D, these two groups had similar APD at 
TABLE 1

Summary of antibodies used in the study

\begin{tabular}{|c|c|c|c|c|c|c|c|c|}
\hline Antibody Name & $\begin{array}{l}\text { Commercial } \\
\text { Source }\end{array}$ & Cat\# & Species & Epitope & Isotype & RRID & $\begin{array}{l}\text { Diluting } \\
\text { Buffer }\end{array}$ & Dilution \\
\hline Connexin 43 & Sigma-Aldrich & C6219 & rabbit polyclonal & $\begin{array}{l}\text { amino acid residues } 363-382 \\
\text { of Connexin } 43\end{array}$ & IgG & AB_476857 & $5 \%$ milk & $1: 1000$ \\
\hline Nav1.5 & $\begin{array}{l}\text { Alomone } \\
\text { Laboratories }\end{array}$ & asc-005 & rabbit polyclonal & $\begin{array}{l}\text { amino acid residues } 17 / 19 \\
\text { of SCN5A }\end{array}$ & IgG & AB_2040001 & $5 \%$ milk & $1: 200$ \\
\hline Kv11.1 & Santa Cruz & sc-377388 & mouse monoclonal & amino acid $96-120$ of HERG & IgG1 & none & $5 \%$ milk & $1: 200$ \\
\hline Kv7.1 & Santa Cruz & sc-20816 & rabbit polyclonal & amino acid $547-676$ of KCNQ1 & IgG & AB_2131551 & $5 \%$ milk & $1: 1000$ \\
\hline GAPDH & EMD Millipore & MAB374 & mouse monoclonal & $\begin{array}{l}\text { glyceraldehyde-3-phosphate } \\
\text { dehydrogenase from rabbit } \\
\text { muscle }\end{array}$ & IgG1 & AB_2107445 & $5 \%$ milk & 1:500 \\
\hline $\begin{array}{l}\text { IRDye } 800 \mathrm{CW} \text { goat } \\
\text { anti-mouse IgG }\end{array}$ & LI-COR & $925-32210$ & goat & IgG & IgG & none & $5 \%$ milk & $1: 10,000$ \\
\hline $\begin{array}{l}\text { IRDye } 800 \mathrm{CW} \text { goat } \\
\text { anti-rabbit IgG }\end{array}$ & LI-COR & $925-32211$ & goat & $\operatorname{IgG}$ & IgG & none & $5 \%$ milk & $1: 10,000$ \\
\hline $\begin{array}{l}\text { IRDye } 680 \mathrm{RD} \text { goat } \\
\text { anti-mouse IgG }\end{array}$ & LI-COR & $925-68070$ & goat & $\operatorname{IgG}$ & IgG & none & $5 \%$ milk & $1: 10,000$ \\
\hline $\begin{array}{l}\text { IRDye } 680 R D \text { goat } \\
\text { anti-rabbit IgG }\end{array}$ & LI-COR & $925-68071$ & goat & IgG & IgG & none & $5 \%$ milk & $1: 10,000$ \\
\hline
\end{tabular}

$80 \%$ repolarization, but $\mathrm{APD}_{30}$ became longer, and repolarization rate (reciprocal of triangulation) became faster after chronic ISO treatment.

To determine whether the increasing in $\mathrm{CV}$ by chronic $\beta$-adrenergic receptor stimulation was associated with a regulation on the transcriptional or translational level, we compared the mRNA and protein expression of specific molecules between control and chronic ISO treatment hESC-CMs. As shown in Fig. 2A, we measured the mRNA expression using qRT-PCR, and GJA1, SCN5A, and Calcium Channel, Voltage-Dependent, L Type, alpha 1C Subunit (CACAN1C) expressions of hESC-CMs were significantly increased after stimulation with $1 \mu$ mol ISO for 24 hours. In the protein level, Fig. 2, B and $\mathrm{C}$ show the Connexin 43 and Nav1.5 protein contents were higher in hESC-CMs with chronic ISO treatment, whereas Kv11.1 (subunit of IKr channel) and Kv7.1 (subunit of IKs channel) level did not differ between control and chronic ISO treatment hESC-CMs.

Next, we evaluated the effect of acute $\beta$-AR stimulation on $\mathrm{CV}$ and APs in hESC-CM monolayers by treatment of the monolayers with $1 \mu \mathrm{mol}$ ISO for 30 minutes. As shown in Fig. 3, A and B, no significant difference of CV was observed before and after acute ISO treatment $(8.4 \pm 0.5 \mathrm{~cm} / \mathrm{s}$ vs. $8.7 \pm 0.6 \mathrm{~cm} / \mathrm{s}$ at $1 \mathrm{~Hz}, P=0.677$ ). Meanwhile, the action
A

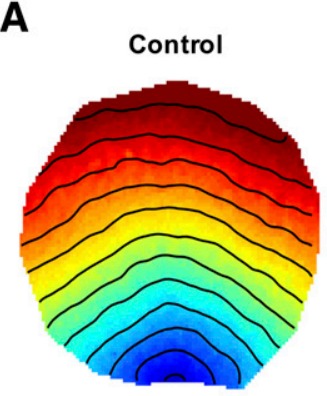

Chronic $\beta$-adrenergic Stimulation

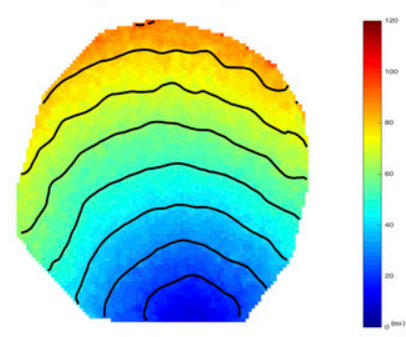

C

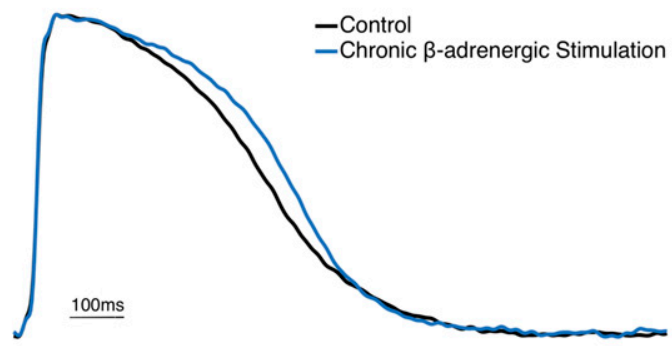

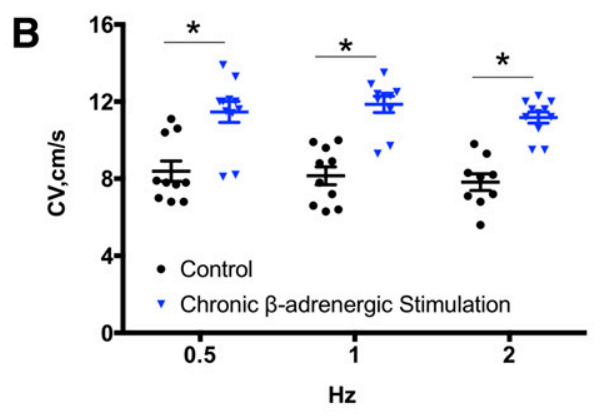

D

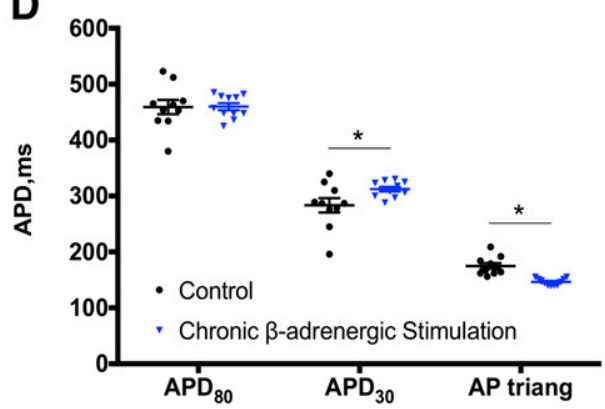

Fig. 1. Comparison of electrophysiological properties of H9 hESC-CM monolayers before and after chronic $\beta$-adrenergic stimulation. (A) Representative activation map. (B) Average conduction velocity at pacing rate between $0.5,1$, and $2 \mathrm{~Hz}, n=10$, two-tailed unpaired $t$ test, $* P<0.05$, significantly different as indicated. (C) Action potential traces for 1-Hz pacing. (D) APD distribution of the monolayers paced at 1 Hz, $n=10$, two-tailed unpaired $t$ test, $* P<0.05$, significantly different as indicated. $\mathrm{APD}_{80}, \mathrm{APD}$ at $80 \%$ repolarization; $\mathrm{AP}$ triang, time from $\mathrm{APD}_{30}$ to $\mathrm{APD}_{80}$. 
A

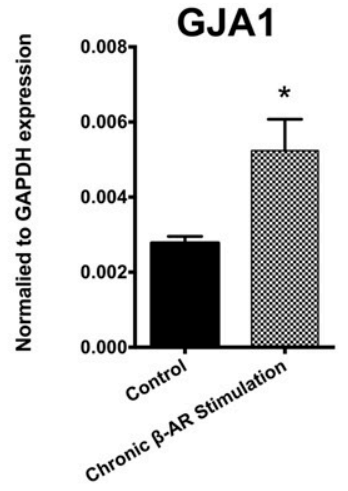

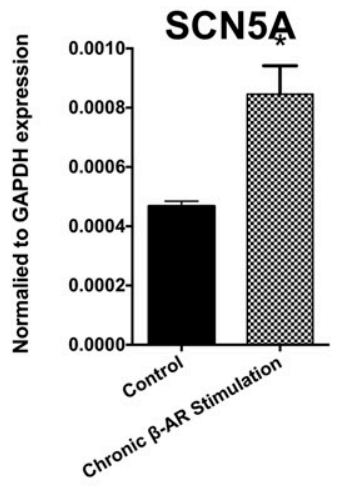
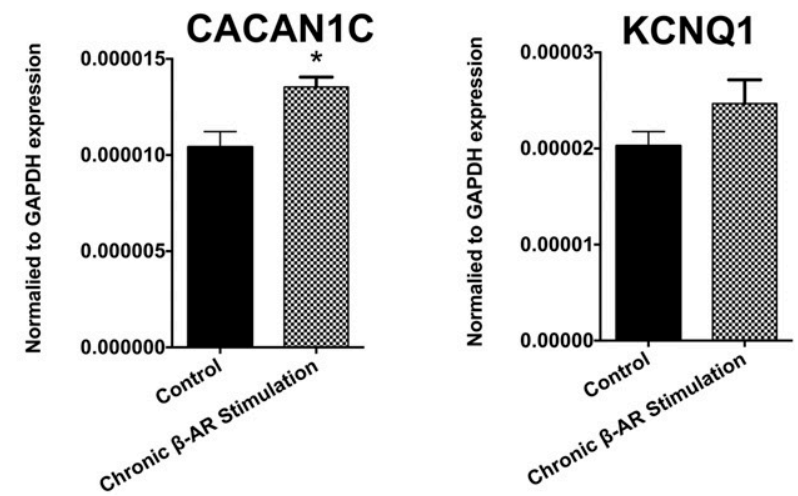

B

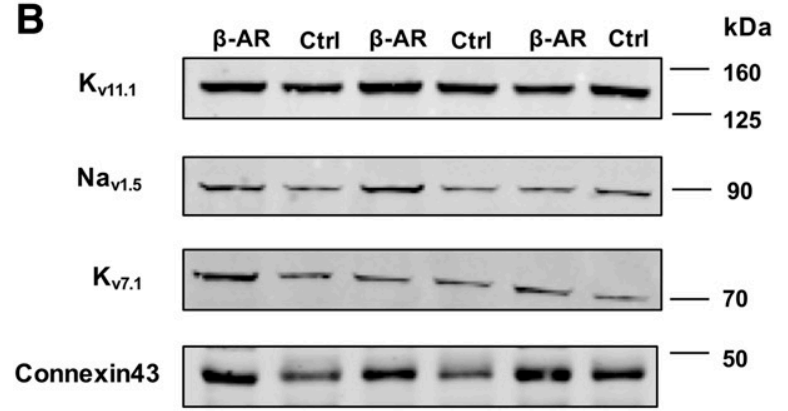

GAPDH

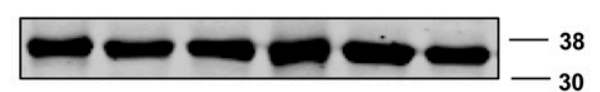

C

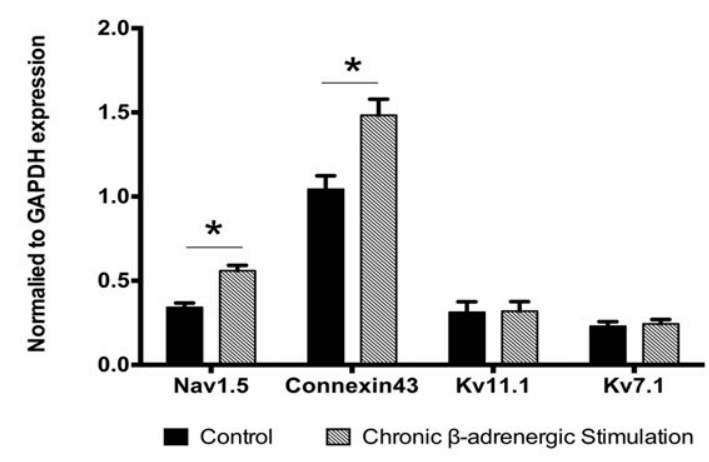

Fig. 2. Comparison of channel and junction proteins expression of hESC-CM monolayers with and without chronic $\beta$-adrenergic stimulation. (A) GJA1, SCN5A, CACAN1C, and KCNQ1 gene expressions normalized to GAPDH, $n=8$ for each gene, two-tailed unpaired $t$ test, $* P<0.05$, significantly different as indicated; (B) Nav1.5, Connexin 43, Kv11.1, Kv7.1, and GAPDH protein expressions; (C) Relative protein (Nav1.5, Connexin 43, Kv11.1, Kv7.1) expressions normalized to GAPDH, $n=6$ for each protein, two-tailed unpaired $t$ test, ${ }^{*} P<0.05$, significantly different as indicated. Ctrl, control.

potentials of these two groups at 1-Hz pacing rate are similar, which are illustrated in Fig. 3, C and D.

$\alpha$-AR Stimulation on CV and APs in hESC-CM Monolayers. To confirm whether the observed AR effects on $\mathrm{CV}$ and $\mathrm{AP}$ appeared exclusively under $\beta$-AR stimulation, analog experiments were performed with hESC-CM monolayers treated with $10 \mu \mathrm{mol} / \mathrm{L} \mathrm{PE}$ for 24 hours as chronic treatments and 30 minutes as acute ones. In contrast to ISO, $\mathrm{PE}$ has no significant effect in altering CV. As shown in Fig. 4, $\mathrm{A}$ and $\mathrm{B}$, there were no significant difference of $\mathrm{CV}$ among chronic $\alpha$-AR stimulation, acute $\alpha$-AR stimulation, and control groups $(8.8 \pm 0.4 \mathrm{~cm} / \mathrm{s}$ vs. $8.7 \pm 0.6 \mathrm{~cm} / \mathrm{s}$ vs. $9.3 \pm 0.4 \mathrm{~cm} / \mathrm{s}$ at $1 \mathrm{~Hz}, P=0.899$ and 0.311$)$. Furthermore, upon acute $\alpha$-AR stimulation, no alterations were detected in action potentials, but chronic $\alpha$-AR stimulation shortened the $\mathrm{APD}_{30}(263 \pm$ 21 milliseconds vs. $214 \pm 40$ milliseconds, $P=0.049)$ and increased the APD triangulation $(145 \pm 6$ milliseconds vs. $161 \pm 10$ milliseconds, $P=0.015$ ) (Fig. 4, C and D).

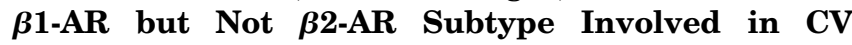
Regulation in hESC-CMs. To further clarify which subtype of $\beta$-AR is responsible for the $\mathrm{CV}$ and AP changes, hESC-CM monolayers were pretreated with selective $\beta 1$-AR inhibitor CGP $20712 \mathrm{~A}(0.3 \mu \mathrm{mol} / \mathrm{L})$ or $\beta 2$-AR inhibitor ICI 118551 $(50 \mathrm{nmol} / \mathrm{L})$ before stimulation with ISO. The working concentration of these two inhibitors was according to published literatures (Hakuno et al., 2002; Chakir et al., 2003). As shown in Fig. 5A, the increase of CV after ISO admission was significantly attenuated in the presence of CGP 20712A $(10.8 \pm 0.2 \mathrm{~cm} / \mathrm{s}$ vs. $8.3 \pm 0.4 \mathrm{~cm} / \mathrm{s}, P=0.001)$ but not in the presence of ICI $118551(10.8 \pm 0.2 \mathrm{~cm} / \mathrm{s}$ vs. $10.3 \pm 0.3 \mathrm{~cm} / \mathrm{s}, P=$ 0.145) (Fig. 5B). Consistent with the previous results, chronic ISO treatment made $\mathrm{APD}_{30}$ longer and $\mathrm{AP}$ triangle lower, but only CGP 20712A attenuated these effects (Fig. 5, C and D).

To further characterize how $\beta 1$-AR and $\beta 2$-AR are involved in responding to modulation of CV and APs in hESC-CM monolayers, we examined the gene expression profiles of GJA1, SCN5A, Kv11.1, and CANAN1C. Twenty-four hours ISO treatment increased the expression of GJA1, SCN5A, and CANAN1C, which were consistent with the previous results. Interestingly, the upregulation of GJA1 and SCN5A by chronic $\beta$-AR stimulation was only abolished by preincubation with CGP 20712A (Fig. 6A). At the protein level, the results were similar with the gene level: ISO increased Connexin (Cx) 43 and Nav1.5 expression, whereas preexposure to CGP 20712A had no effect (Fig. 6B).

B-AR Stimulation Increasing CV of hESC-CMs by Upregulation of Cx43 Expression via the Protein Kinase A/Mitogen-Activated Protein Kinase-Extracellular Signal-Regulated Kinase/Mitogen-Activated Protein Kinase Pathway. To further investigate the potential mechanism and signal pathway of ISO-induced CV increase, hESC-CM monolayers were incubated with ISO alone or pretreated, respectively, with $10 \mathrm{mmol} / \mathrm{L} \mathrm{H} 89$ [specific inhibitor of protein kinase A (PKA)], $20 \mu \mathrm{mol} / \mathrm{L}$ SB203580 [specific inhibitor of mitogenactivated protein kinase (MAPK)], or $20 \mu \mathrm{mol} / \mathrm{L}$ PD98059 [specific inhibitor of MAPK/extracellular signal-regulated kinase (MEK)] for 1 hour before ISO admission as previously described. As shown in Fig. 7, A and B, coadministration 
A

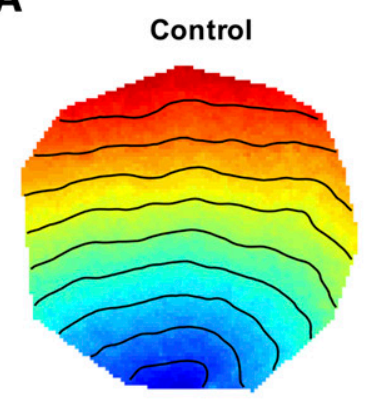

Acute $\beta$-adrenergic Stimulation

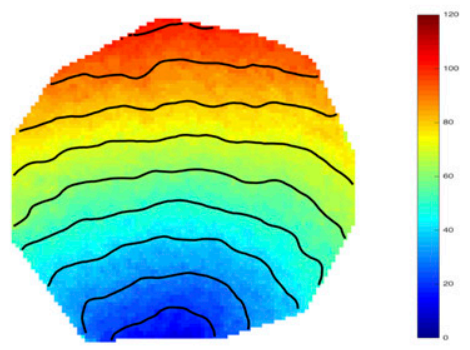

B

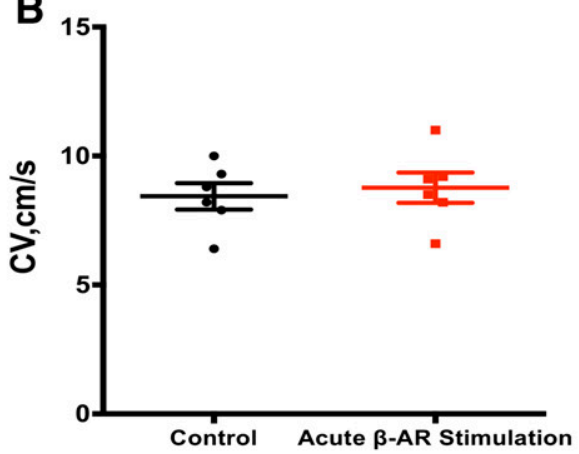

D

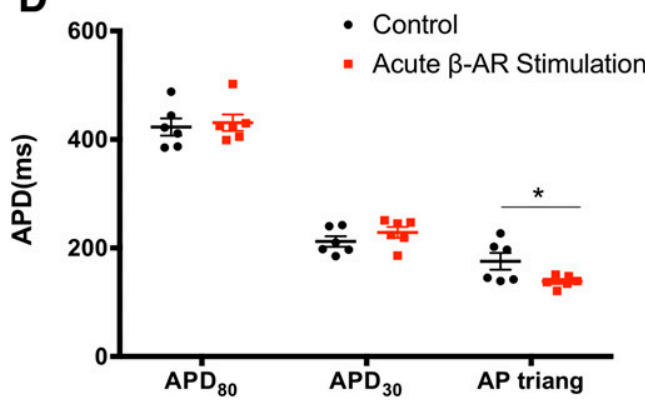

Fig. 3. Comparison of electrophysiological properties of H9 hESC-CM monolayers before and after acute $\beta$-adrenergic stimulation. (A) Representative activation map. (B) Average conduction velocity at $1-\mathrm{Hz}$ pacing rate, $n=6$, two-tailed unpaired $t$ test, $* P<0.05$, significantly different as indicated. (C) Action potential traces for 1-Hz pacing. (D) APD distribution of the monolayers paced at $1 \mathrm{~Hz}, n=6$, two-tailed unpaired $t$ test, $* P<0.05$, significantly different as indicated. $\mathrm{APD}_{80}, \mathrm{APD}$ at $80 \%$ repolarization; $\mathrm{AP}$ triang, time from $\mathrm{APD}_{30}$ to $\mathrm{APD}_{80}$.

of H89/SB203580/PD98059 with ISO could attenuate the effect to increase $\mathrm{CV}$ by $\beta$-AR stimulation. In addition, the AP change caused by $\beta$-AR stimulation was also suppressed by these three specific inhibitors (Fig. 7C), and the prolongation of $\mathrm{APD}_{30}$ and acceleration of repolarization rate by ISO was restained in the presence of H89, SB203580, or PD98059 (Fig. 7D). Again, $\beta$-AR stimulation or inhibition had little effect on APD at $80 \%$ repolarization. Importantly, inhibition of PKA/MEK/MAPK signaling prevented the increased expression of $\mathrm{Cx} 43$ by ISO (Fig. 8A) but had no effect on expression of Nav1.5
A
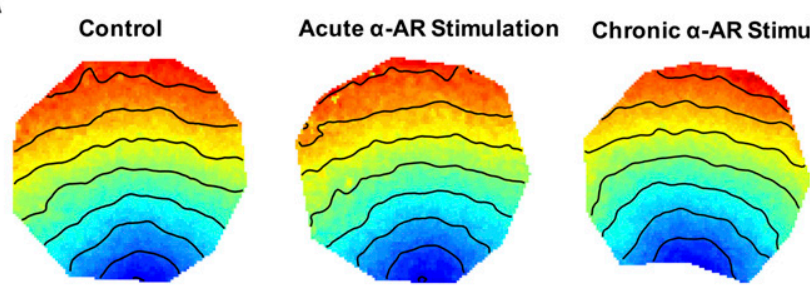

C

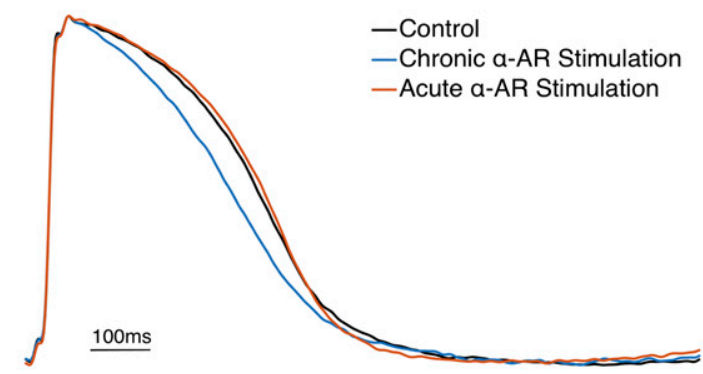

B

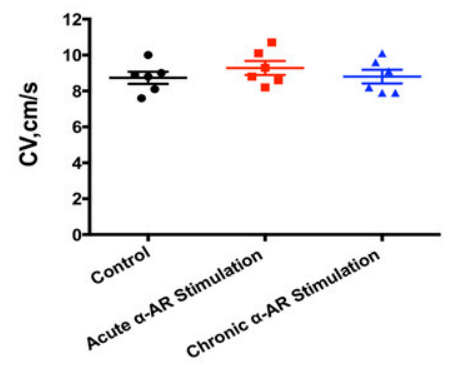

D

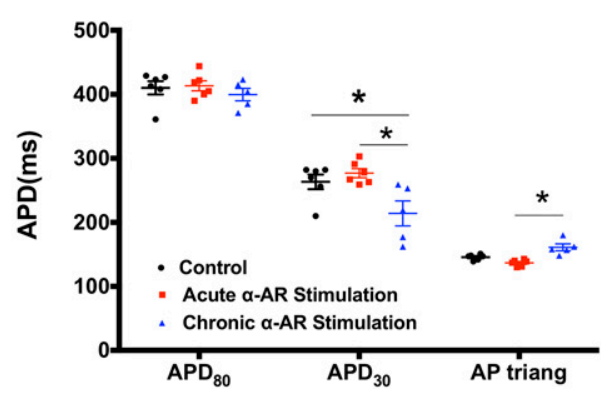

Fig. 4. Comparison of electrophysiological properties of H9 hESC-CM monolayers before and after acute or chronic $\alpha$-AR stimulation. (A) Representative activation map. (B) Average conduction velocity at $1-\mathrm{Hz}$ pacing rate, $n=6$ for each group, one-way ANOVA followed by Tukey's post hoc tests, $* P<0.05$, significantly different as indicated. (C) Action potential traces for $1-\mathrm{Hz}$ pacing. (D) APD distribution of the monolayers paced at $1 \mathrm{~Hz}$, $n=6$ for each group, one-way ANOVA followed by Tukey's post hoc tests, $* P<0.05$, significantly different as indicated. APD ${ }_{80}$, APD at $80 \%$ repolarization; $\mathrm{AP}$ triang, time from $\mathrm{APD}_{30}$ to $\mathrm{APD}_{80}$. 

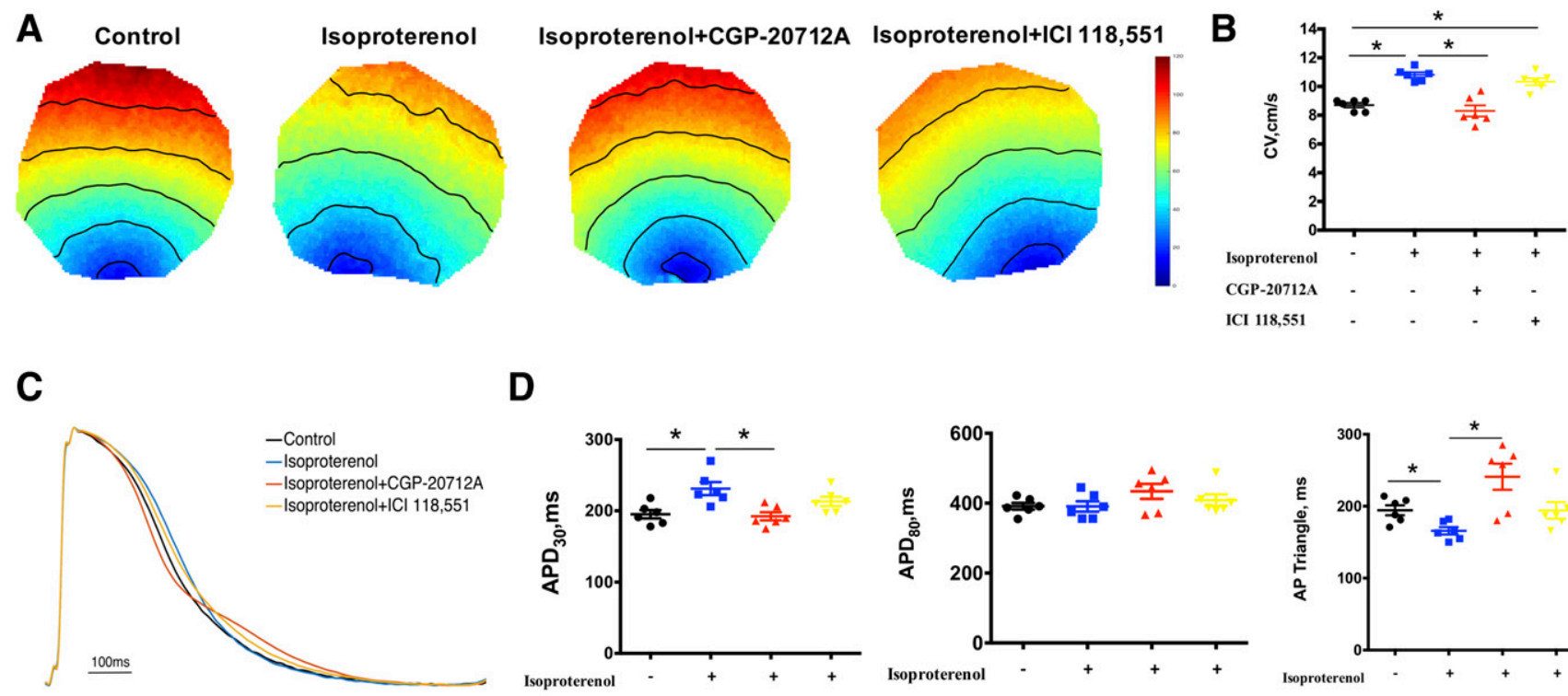

D
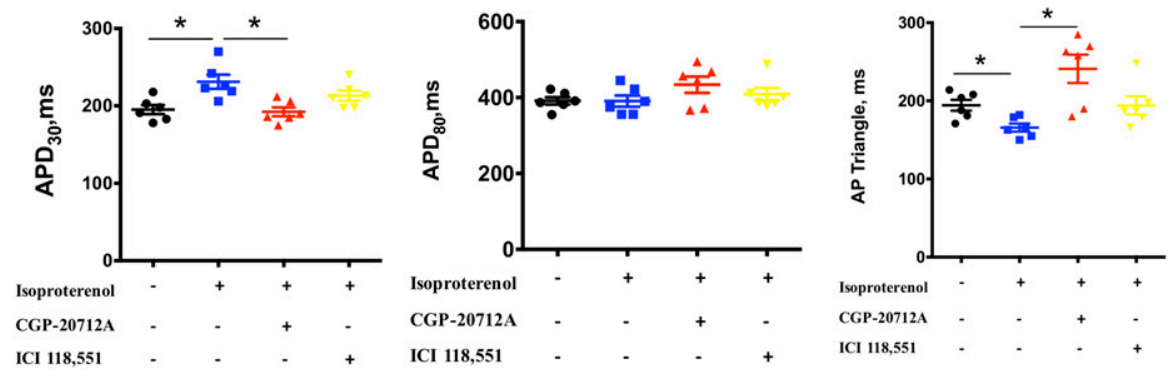

Fig. 5. Comparison of electrophysiological properties of H9 hESC-CM monolayers between control, Isoproterenol, Isoproterenol + CGP 20712A, and Isoproterenol + ICI 118551 groups. (A) Representative activation map. (B) Average conduction velocity at 1-Hz pacing rate, $n=6$ for each group, one-way ANOVA followed by Tukey's post hoc tests, ${ }^{*} P<0.05$, significantly different as indicated. (C) Action potential traces for $1-\mathrm{Hz}$ pacing. (D) APD distribution of the monolayers paced at $1 \mathrm{~Hz}, n=6$ for each group, one-way ANOVA followed by Tukey's post hoc tests, ${ }^{*} P<0.05$, significantly different as indicated. $\mathrm{APD}_{80}, \mathrm{APD}$ at $80 \%$ repolarization; $\mathrm{AP}$ triang, time from $\mathrm{APD}_{30}$ to $\mathrm{APD}_{80}$.

(Fig. 8B), suggesting PKA/MEK/MAPK pathway activated by $\beta$-AR stimulation only contributes to the regulation of Cx43 expression.

\section{Discussion}

hESC-CMs have emerged as the prototypical source for applications in disease modeling and drug screening differentiation (Pal, 2009; Vidarsson et al., 2010). However, such applications require hESC-CMs to faithfully recapitulate the physiology of authentic adult cells, especially the electrophysiological properties (Liu et al., 2016). Previous studies of authentic cardiomyocytes revealed that ARs play a critical role in the regulation of the electrophysiological performance of cardiomyocytes, such as the conduction velocity and the action potential (Campbell et al., 2014). In this study, we investigated the regulation effect of ARs in hESC-CMs' electrophysiological characters and demonstrated that 1) $\beta$-AR stimulation in hESC-CM monolayers for 24 hours increased CV by approximately $50 \%$, which did not occur after $\alpha$-AR stimulation; 2) $\beta 1$-ARs, not $\beta 2$-ARs, were involved in the modulation of CV in hESC-CM monolayers; and 3) $\beta 1$-AR stimulation induced marked alterations in the expression of Connexins 43 and SCN5A, which fits well with the increased $\mathrm{CV}(4) \beta 1-\mathrm{AR}$ stimulation upregulated $\mathrm{Cx} 43$ expression via PKA/MEK/MAPK pathway.

The gap junction protein Connexins play an important role in regulation of CV (Campbell et al., 2014). In human heart, the main isoforms of Connexins expressed are $\mathrm{Cx} 43, \mathrm{Cx} 40$, $\mathrm{Cx} 37$, and $\mathrm{Cx} 45$, and $\mathrm{Cx} 43$ is the most abundant one (Manring et al., 2018). Radioactive labeling tests have demonstrated that half-life of $\mathrm{Cx} 43$ is only 1 to 2 hours, which allows dynamic regulation of its expression and further affects the CV (Leithe and Rivedal, 2007). Considerable evidence proved that Connexin expression could be altered by $\beta$-AR stimulation in animal models and primary cardiomyocytes. In adult rats, 24 hours continuous stimulation with isoprenaline infusion in vivo resulted in an upregulation of cardiac $\mathrm{Cx} 43$ expression, whereas $\mathrm{Cx} 40$ remained unchanged (Salameh et al., 2006). Increases in CV $(\sim 25 \%)$ and $\mathrm{Cx} 43$ expression in response to 24 hours incubation with isoprenaline were observed in neonatal rat cardiac myocytes (de Boer et al., 2007). Changes in expression of Cx43 only occurred after long exposures to isoprenaline, whereas some studies reported no $\mathrm{CV}$ increase to acute $\beta$-AR stimulation (Salameh and Dhein, 2011). In the present study, we found CV among hESC-CM monolayers could be increased by $\sim 50 \%$ after 24 hours incubation with isoprenaline, whereas no response was observed with exposure to isoprenaline for 10 minutes. Moreover, the Western blot and qRT-PCR analysis confirmed that expression of $\mathrm{Cx} 43$ and GJA1 gene was increased by $\sim 1.5$ times and $\sim 1.8$ times after chronic $\beta$-AR stimulation, respectively.

To further understand the role of different $\beta$-AR subtypes on $\mathrm{Cx} 43$ expression and CV regulation, the hESC-CMs were pretreated with selective $\beta 1$ - or $\beta 2$-AR inhibitor. We found the effect of isoprenaline on CV was only antagonized by $\beta 1$-AR inhibitor not $\beta 2$-AR inhibitor. This is inconsistent with the results of a previous study, which suggested $\beta 2$-AR mediates the upregulation of nonphosphorylated $\mathrm{Cx} 43$ in neonatal rat cardiac myocytes (Xia et al., 2009). One important issue that needs to be clarified is whether $\beta 2$-AR been well-expressed in the hESC-CMs with d40-45 postdifferentiation, since the expression level of ARs has been proven to depend on the culture time. Literature confirmed that $\beta$-AR regulates beating rate and contractile responses in hESC-CMs with d29 to $\mathrm{d} 79$ after differentiation, and both $\beta 1$ - and $\beta 2$-AR genes have been detected by qRT-PCR. Moreover, the researchers found the increased beating rate after isoprenaline treatment was significantly reduced in the presence of $\beta 2$-AR inhibitor 
A GJA1

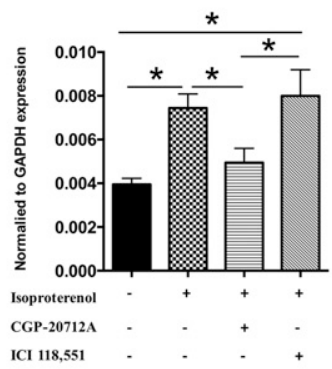

SCN5A

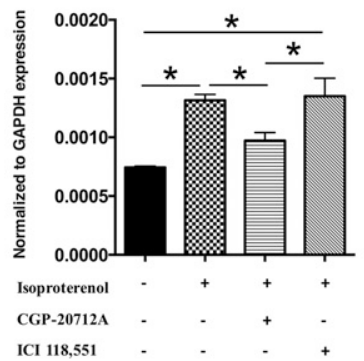

CACAN1C

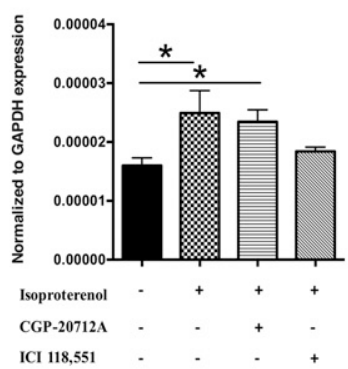

KCNQ1

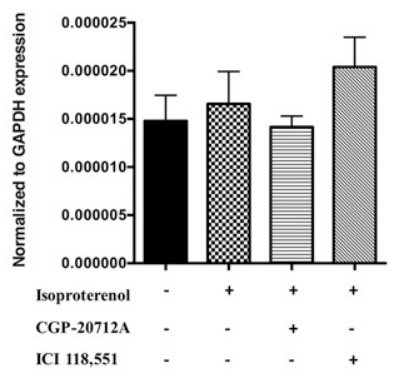

B
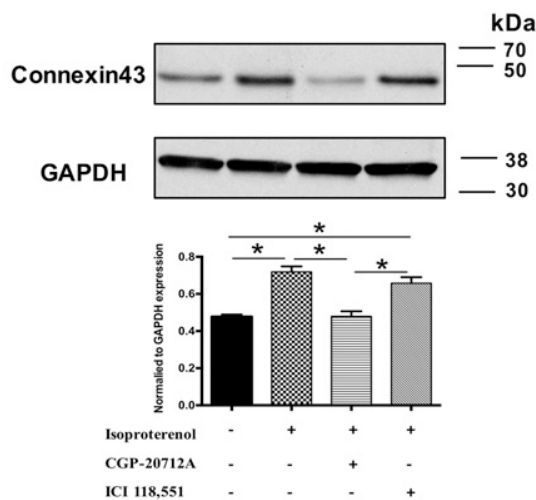
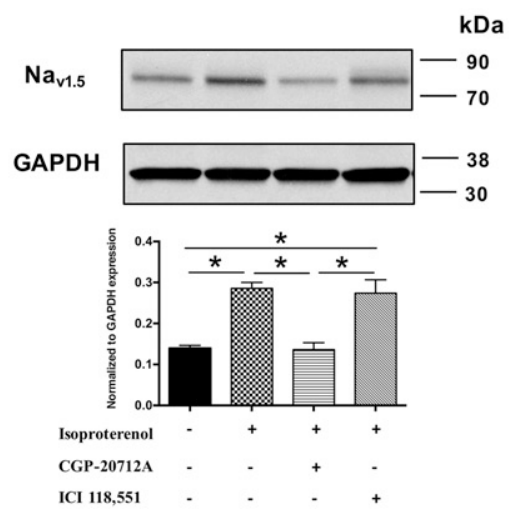

Fig. 6. Comparison of channel and junction proteins expression of hESC-CM monolayers between control, Isoproterenol, Isoproterenol + CGP 20712A, and Isoproterenol + ICI 118551 groups. (A) GJA1, SCN5A, CACAN1C, and KCNQ1 gene expressions normalized to GAPDH, $n=8$ for each gene, one-way ANOVA followed by Tukey's post hoc tests, $* P<0.05$, significantly different as indicated; (B) Nav1.5 and Connexin 43 protein expressions normalized to GAPDH, $n=5$ for Nav1.5 and $n=6$ for Connexin 43, one-way ANOVA followed by Tukey's post hoc tests, ${ }^{*} P<0.05$, significantly different as indicated.

(Brito-Martins et al., 2008). This can be interpreted as $\beta 2-\mathrm{AR}$ existing in hESC-CMs during this period. Therefore, it is reasonable to assume that $\beta 2-\mathrm{AR}$ was expressed in hESC-CMs with $\mathrm{d} 40-45$ postdifferentiation but did not participate in the CV regulation.
Cx43 can be phosphorylated by PKA, protein kinase C, protein kinase G, and MAPK (Marquez-Rosado et al., 2012 ). $\beta 1$-ARs can activate adenylyl cyclase to product cAMP (Fu et al., 2014). As the direct substrate of cAMP, PKA has been implicated in the various biologic responses of $\beta 1$-ARs
A

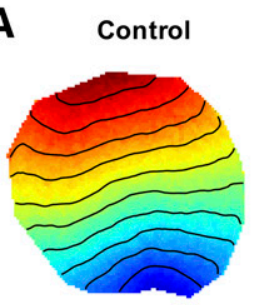

Isoproterenol

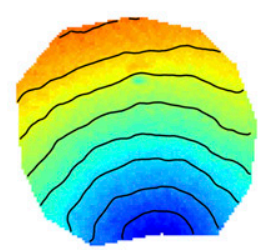

Isoproterenol+H89

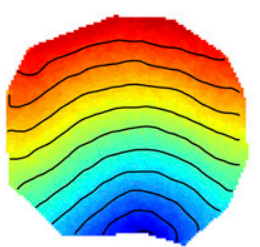

Isoproterenol+SB203580 Isoproterenol+PD98059
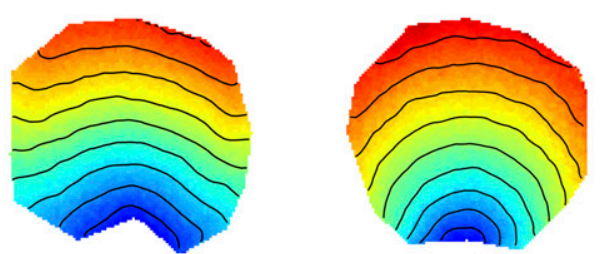

D

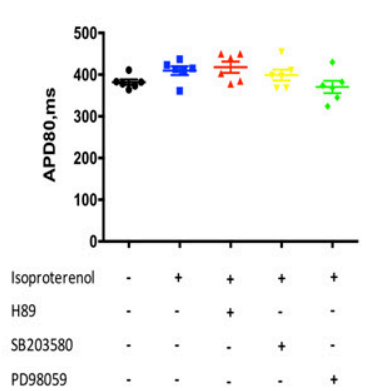

B

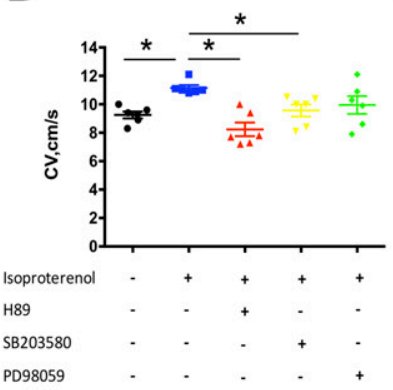

C

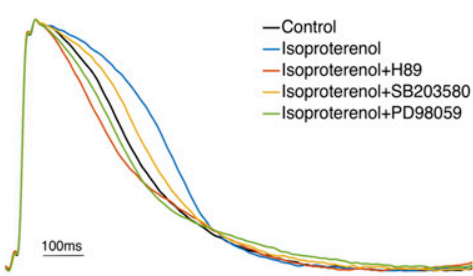

Fig. 7. Comparison of electrophysiological properties of H9 hESC-CM monolayers between control, Isoproterenol, Isoproterenol + H89, Isoproterenol + SB203580, and Isoproterenol + PD98059 groups. (A) Representative activation map. (B) Average conduction velocity at $1-\mathrm{Hz}$ pacing rate, $n=6$ for each group, one-way ANOVA followed by Tukey's post hoc tests, ${ }^{*} P<0.05$, significantly different as indicated. (C) Action potential traces under 1-Hz pacing. (D) APD distribution of the monolayers paced at $1 \mathrm{~Hz}, n=6$ for each group, one-way ANOVA followed by Tukey's post hoc tests, ${ }^{*} P<0.05$, significantly different as indicated. $\mathrm{APD}_{80}, \mathrm{APD}$ at $80 \%$ repolarization. 
A
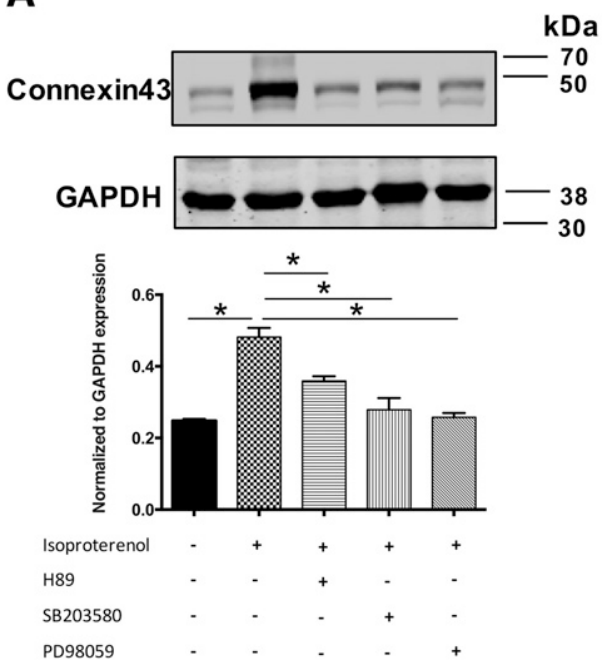

B
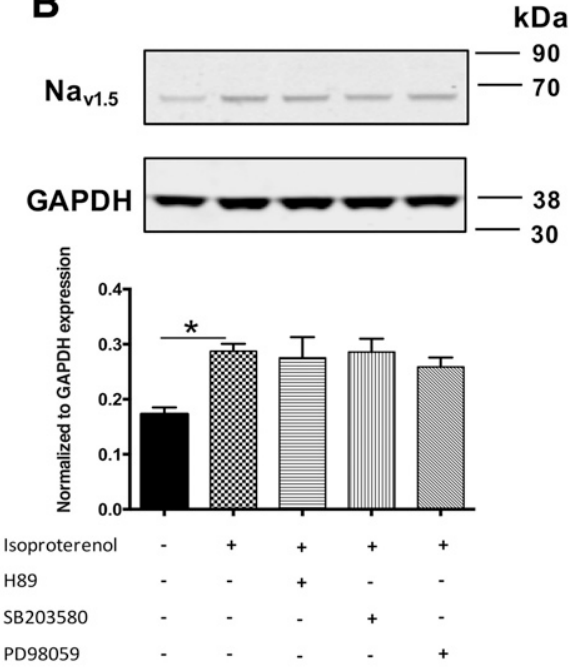

Fig. 8. Comparison of channel and junction proteins expression of hESC-CM monolayers between control, Isoproterenol, Isoproterenol + H89, Isoproterenol + SB203580, and Isoproterenol + PD98059 groups. (A) Connexin 43 expressions normalized to GAPDH, $n=6$, one-way ANOVA followed by Tukey's post hoc tests, $* P<0.05$, significantly different as indicated; (B) Nav1.5 expressions normalized to GAPDH, $n=5$, one-way ANOVA followed by Tukey's post hoc tests, $* P<$ 0.05 , significantly different as indicated.
(Saad et al., 2018). Our study demonstrated that effect of $\beta 1-\mathrm{AR}$ on increasing $\mathrm{CV}$ and regulating $\mathrm{Cx} 43$ expression was driven via PKA/MEK/MAPK pathway in hESC-CMs because H89-PKA inhibitor, SB203580-MAPK inhibitor, and PD98059MEK inhibitor could attenuate the above-mentioned effect. Consistent with this, several studies have shown that activation of the cAMP/PKA pathway can regulate $\mathrm{Cx} 43$ expression and a downstream activation of MEK, and MAPK was dependent on PKA after isoprenaline in adult rats (Zhang et al., 2005) or in neonatal rat cardiomyocyte (Krishnamurthy et al., 2007).

The other factor that plays a role in modulation of $\mathrm{CV}$ is the upstroke of the AP (Perry and Illsley, 1986). The sodium channels contribute to the AP upstroke, whereas the magnitude of $\mathrm{I}_{\mathrm{Na}}$ can be increased by $\beta$-AR activation(Frohnwieser et al., 1997; Lu et al., 1999) (Frohnwieser et al., 1997; Lu et al., 1999). Another study demonstrated that the expression of $\mathrm{Na}_{\mathrm{v} 1.5}$ is upregulated via CaMKII activation after $\beta$-AR stimulation (Jost et al., 2005). Therefore, the effects of $\beta$-AR on the AP upstroke are a combination of its effects on increasing the magnitude of $\mathrm{I}_{\mathrm{Na}}$ and upregulating sodium channel expression. However, some studies indicated the predicted maximal effect of $\mathrm{I}_{\mathrm{Na}}$ on $\mathrm{CV}$ is an increase of only $4 \%$ to $5 \%$ after $\beta$-AR stimulation (Boyle et al., 2019). Nevertheless, we believed the sodium channel was not the main determinant in modulation of $\mathrm{CV}$ by $\beta$-AR stimulation in hESC-CMs. Although optical action potentials used in our experiment could not provide direct measurements of individual sodium currents or AP upstroke, the Western blot and qRT-PCR analysis confirmed that expression of Nav1.5 and SCN5A increased $\sim 1.5 \times$ and $\sim 2 \times$ times after chronic $\beta$-AR stimulation.

$\beta$-AR signaling also influences AP duration. The slowly activating delayed rectifier potassium channel $\left(\mathrm{I}_{\mathrm{Ks}}\right)$ can be affected by $\beta$-AR stimulation via PKA pathway (Jost et al., $2007)$. In the present, we observed a significant prolongation of $\mathrm{ADP}_{30}$ in hESC-CMs after $\beta$-AR stimulation, which is discordant with the findings of other prior studies. In large mammals, $\beta$-AR stimulation increases the magnitude of $\mathrm{I}_{\mathrm{Ks}}$ to promote APD shortening (Jost et al., 2005), whereas addition of isoproterenol can increase the steepness of the AP trace and reduces the effective refractory period in the whole hearts $(\mathrm{Ng}$ et al., 2007). The difference of APD change in hESC-CMs by
$\beta$-AR stimulation is maybe due to the dynamic change of major repolarization potassium channels across different stages of differentiation, which was systematically characterized in our prior study; at this phase, the expression of IKs was at a low level and contributed little to APD (Wang et al., 2019).

In heart failure, changes in $\beta$-AR amount and subtypes can lessen the effect of adrenergic regulation on sodium channels and Connexin proteins and attenuate the increase in conduction velocity by $\beta$-AR stimulation, which may contribute to arrhythmia (Spadari et al., 2018). The practical application of human stem cell-derived cardiomyocytes for myocardial regeneration requires a thorough and clear understanding of the adrenoceptor response to reduce their proarrhythmogenic potential. To our knowledge, this is the first research to systematically evaluate the electrophysiological response in hESC-CMs by $\beta$-AR and $\alpha$-AR stimulation in terms of action potential duration and conduction velocity. These findings may enrich the knowledge of hESC-CMs' electrophysiological characteristics.

The study has some limitations. Firstly, since it was subject to the complexity and lengthy time span, we did not conduct the experiments in multiple cell lines. We adopted hESCs to derive cardiomyocytes, since they have been documented as the gold standard in terms of their pluripotency and natural development. Among different hESC lines, the H9 line has been shown to have good cardiomyogenic potential (Sepac et al., 2012). Secondly, our study did not detect the ARs' expression density and investigate the ARs' response in hESC-CMs of different differentiation stages. Notably, the expression and response of $\mathrm{ARs}$ might be associated with the differentiation stage. Thirdly, we used the optical mapping to perform the experiments in single confluent cell monolayer, and this had the advantage of hundreds of measurements from very large populations of cells; however, it could not assess individual ionic currents and $\mathrm{dV} / \mathrm{dt}$, although it had the advantage of hundreds of measurements from very large populations of cells from a single confluent cell monolayer, in which the cells were not affected by patch pipette solution, and their structure remained unperturbed, and could measure net ionic current activity through measurements of the action potential (Herron et al., 2012). Fourthly, we used total protein 
Western blots rather than isolated membrane ones in our study, which may have affected the quantity of the results.

In conclusion, our findings confirm electrophysiological response to AR activation in hESCs-CMs and depict a concise signaling pathway in the ARs regulation of electrical propagation. It is $\beta 1-\mathrm{AR}$ and not $\beta 2-\mathrm{AR}$ contributing to the upregulation of $\mathrm{Cx} 43$ and modification of conduction velocity via $\mathrm{PKA} / \mathrm{MEK} /$ MAPK pathway.

\section{Authorship Contributions}

Participated in research design: Jiang, Wang.

Conducted experiments: Jiang, $\mathrm{Hu}, \mathrm{F}$. Li, G. Li.

Contributed new reagents or analytic tools: Jiang, $\mathrm{Hu}$.

Performed data analysis: Jiang, F. Li.

Wrote or contributed to the writing of the manuscript: Jiang, $\mathrm{Hu}$, Wang.

\section{References}

Bhattacharya S, Burridge PW, Kropp EM, Chuppa SL, Kwok WM, Wu JC, Boheler KR, and Gundry RL (2014) High efficiency differentiation of human pluripotent stem cells to cardiomyocytes and characterization by flow cytometry. J Vis Exp $\mathbf{9 1}$ 52010.

Boyle PM, Franceschi WH, Constantin M, Hawks C, Desplantez T, Trayanova NA and Vigmond EJ (2019) New insights on the cardiac safety factor: unraveling the relationship between conduction velocity and robustness of propagation. J Mol Cell Cardiol 128:117-128.

Brito-Martins M, Harding SE, and Ali NN (2008) beta(1)- and beta(2)-adrenoceptor responses in cardiomyocytes derived from human embryonic stem cells: comparison with failing and non-failing adult human heart. $B r J$ Pharmacol 153:751-759.

Campbell AS, Johnstone SR, Baillie GS, and Smith G (2014) $\beta$-Adrenergic modulation of myocardial conduction velocity: connexins vs. sodium current. $J$ Mol Cell Cardiol 77:147-154.

Chakir K, Xiang Y, Yang D, Zhang SJ, Cheng H, Kobilka BK, and Xiao RP (2003) The third intracellular loop and the carboxyl terminus of beta2-adrenergic receptor confer spontaneous activity of the receptor. Mol Pharmacol 64:1048-1058.

Dakhore S, Nayer B, and Hasegawa K (2018) Human pluripotent stem cell culture: current status, challenges, and advancement. Stem Cells Int 2018:7396905.

de Boer TP, van Rijen HV, Van der Heyden MA, Kok B, Opthof T, Vos MA, Jongsma HJ, de Bakker JM, and van Veen TA (2007) Beta-, not alpha-adrenergic stimulation enhances conduction velocity in cultures of neonatal cardiomyocytes. Circ J 71: 973-981.

Frohnwieser B, Chen LQ, Schreibmayer W, and Kallen G (1997) Modulation of the human cardiac sodium channel alpha-subunit by cAMP-dependent protein kinase and the responsible sequence domain. J Physiol 498:309-318.

Fu Q, Kim S, Soto D, De Arcangelis V, DiPilato L, Liu S, Xu B, Shi Q, Zhang J, and Xiang YK (2014) A long lasting $\beta 1$ adrenergic receptor stimulation of cAMP/ protein kinase A (PKA) signal in cardiac myocytes. J Biol Chem 289:14771-14781.

Hakuno D, Fukuda K, Makino S, Konishi F, Tomita Y, Manabe T, Suzuki Y, Umezawa A, and Ogawa S (2002) Bone marrow-derived regenerated cardiomyocytes (CMG Cells) express functional adrenergic and muscarinic receptors. Circulation 105:380-386.

Herron TJ, Lee P, and Jalife J (2012) Optical imaging of voltage and calcium in cardiac cells \& tissues. Circ Res 110:609-623.

Jost N, Papp JG, and Varró A (2007) Slow delayed rectifier potassium current (IKs) and the repolarization reserve. Ann Noninvasive Electrocardiol 12:64-78.

Jost N, Virág L, Bitay M, Takács J, Lengyel C, Biliczki P, Nagy Z, Bogáts G, Lathrop DA, Papp JG, et al. (2005) Restricting excessive cardiac action potential and QT prolongation: a vital role for IKs in human ventricular muscle. Circulation 112: 1392-1399.

Krishnamurthy P, Subramanian V, Singh M, and Singh K (2007) Beta1 integrins modulate beta-adrenergic receptor-stimulated cardiac myocyte apoptosis and myocardial remodeling. Hypertension 49:865-872.
Leithe E and Rivedal E (2007) Ubiquitination of gap junction proteins. J Membr Biol 217:43-51.

Liu J, Laksman Z, and Backx PH (2016) The electrophysiological development of cardiomyocytes. Adv Drug Deliv Rev 96:253-273.

Lu T, Lee HC, Kabat JA, and Shibata EF (1999) Modulation of rat cardiac sodium channel by the stimulatory G protein alpha subunit. J Physiol 518:371-384.

Manring HR, Dorn LE, Ex-Willey A, Accornero F, and Ackermann MA (2018) At the heart of inter- and intracellular signaling: the intercalated disc. Biophys Rev 10: 961-971.

Márquez-Rosado L, Solan JL, Dunn CA, Norris RP, and Lampe PD (2012) Connexin43 phosphorylation in brain, cardiac, endothelial and epithelial tissues. Biochim Biophys Acta 1818:1985-1992.

Ng GA, Brack KE, Patel VH, and Coote JH (2007) Autonomic modulation of electrical restitution, alternans and ventricular fibrillation initiation in the isolated heart. Cardiovasc Res 73:750-760.

Pal R (2009) Embryonic stem (ES) cell-derived cardiomyocytes: a good candidate for cell therapy applications. Cell Biol Int 33:325-336.

Perry RS and Illsley SS (1986) Basic cardiac electrophysiology and mechanisms of antiarrhythmic agents. Am J Hosp Pharm 43:957-974.

Pillekamp F, Haustein M, Khalil M, Emmelheinz M, Nazzal R, Adelmann R, Nguemo F, Rubenchyk O, Pfannkuche K, Matzkies M, et al. (2012) Contractile properties of early human embryonic stem cell-derived cardiomyocytes: beta-adrenergic stimulation induces positive chronotropy and lusitropy but not inotropy. Stem Cells Dev 21:2111-2121.

Protze SI, Lee JH, and Keller GM (2019) Human pluripotent stem cell-derived cardiovascular cells: from developmental biology to therapeutic applications. Cell Stem Cell 25:311-327.

Saad NS, Elnakish MT, Ahmed AAE, and Janssen PML (2018) Protein kinase A as a promising target for heart failure drug development. Arch Med Res 49:530-537.

Salameh A and Dhein S (2011) Adrenergic control of cardiac gap junction function and expression. Naunyn Schmiedebergs Arch Pharmacol 383:331-346.

Salameh A, Frenzel C, Boldt A, Rassler B, Glawe I, Schulte J, Mühlberg K, Zimmer HG, Pfeiffer D, and Dhein S (2006) Subchronic alpha- and beta-adrenergic regulation of cardiac gap junction protein expression. FASEB $J$ 20:365-367.

Samak M and Hinkel R (2019) Stem cells in cardiovascular medicine: historical overview and future prospects. Cells 8:1530.

Sepac A, Si-Tayeb K, Sedlic F, Barrett S, Canfield S, Duncan SA, Bosnjak ZJ, and Lough JW (2012) Comparison of cardiomyogenic potential among human ESC and iPSC lines. Cell Transplant 21:2523-2530.

Spadari RC, Cavadas C, de Carvalho AETS, Ortolani D, de Moura AL, and Vassalo PF (2018) Role of beta-adrenergic receptors and sirtuin signaling in the heart during aging, heart failure, and adaptation to stress. Cell Mol Neurobiol 38: $109-120$.

van den Heuvel NH, van Veen TA, Lim B, and Jonsson MK (2014) Lessons from the heart: mirroring electrophysiological characteristics during cardiac development to in vitro differentiation of stem cell derived cardiomyocytes. J Mol Cell Cardiol 67: $12-25$.

Veeraraghavan R, Gourdie RG, and Poelzing S (2014) Mechanisms of cardiac conduction: a history of revisions. Am J Physiol Heart Circ Physiol 306: H619-H627.

Vidarsson H, Hyllner J, and Sartipy P (2010) Differentiation of human embryonic stem cells to cardiomyocytes for in vitro and in vivo applications. Stem Cell Rev Rep 6:108-120.

Wang Y, Zhu R, and Tung L (2019) Contribution of potassium channels to action potential repolarization of human embryonic stem cell-derived cardiomyocytes. $B r$ $J$ Pharmacol 176:2780-2794.

Xia Y, Gong KZ, Xu M, Zhang YY, Guo JH, Song Y, and Zhang P (2009) Regulation of gap-junction protein connexin 43 by beta-adrenergic receptor stimulation in rat cardiomyocytes. Acta Pharmacol Sin 30:928-934.

Zhang GX, Kimura S, Nishiyama A, Shokoji T, Rahman M, Yao L, Nagai Y, Fujisawa Y, Miyatake A, and Abe Y (2005) Cardiac oxidative stress in acute and chronic isoproterenol-infused rats. Cardiovasc Res 65:230-238.

Address correspondence to: Yin Wang, Department of Cardiovascular Surgery, Union Hospital, Tongji Medical College, Huazhong University of Science and Technology, 1277 Jiefang Ave., Wuhan, Hubei, 430022, China. E-mail: wangyin0817@hotmail.com 